\title{
Is Sleep Timing Related to Objectively Measured Physical Activity and Sedentary Behavior in Older Women?
}

\author{
Wan-Chi Huang (D) \\ Chia-Shuan Chang (D) ${ }^{2}$ \\ Chien-Yu Lin ${ }^{3}$ \\ Ting-Fu Lai' \\ Ming-Chun Hsueh ${ }^{4}$ \\ Yung Liao (D) ${ }^{1,5}$ \\ Jong-Hwan Park (1D ${ }^{6}$ \\ 'Department of Health Promotion and \\ Health Education, National Taiwan \\ Normal University, Taipei, 106, Taiwan; \\ ${ }^{2}$ Institute of Health Behaviors and \\ Community Sciences, National Taiwan \\ University, Taipei, 100, Taiwan; ${ }^{3}$ Graduate \\ School of Sport Sciences, Waseda \\ University, Tokorozawa City, Saitama \\ Prefecture, 359-I 192, Japan; ${ }^{4}$ Graduate \\ Institute of Sport Pedagogy, University of \\ Taipei, Taipei City, III53, Taiwan; \\ ${ }^{5}$ Faculty of Sport Sciences, Waseda \\ University, Tokorozawa City, Saitama, \\ 359-I 192, Japan; ${ }^{6} \mathrm{Health}$ Convergence \\ Medicine Laboratory, Biomedical \\ Research Institute, Pusan National \\ University Hospital, Busan, 4924I, Korea
}

Correspondence: Jong-Hwan Park Health Convergence Medicine Laboratory, Biomedical Research Institute, Pusan National University

Hospital, Busan, 4924I, Korea

$\mathrm{Tel} / \mathrm{Fax}+82-5 \mathrm{I}-240-707 \mathrm{I}$

Email parkj@pusan.ac.kr
Purpose: Sleep, sedentary behavior, and physical activity are the components of the 24-hour model, while the timing of sleep may play a critical role to impact waking behaviors. Therefore, the present study aimed to examine the relationship of sleep timing with sedentary behavior, light-intensity, moderate-to-vigorous-intensity physical activity, and daily steps in older women.

Patients and Methods: A total of 90 community-dwelling older women (70.5 \pm 5.4 years) were included in our sample. Multiple linear regression models were used to estimate the associations of accelerometer-measured sedentary behavior and physical activity metrics with the three sleep timing indicators (bedtime and wake time, and mid-sleep time). Bedtime and wake time were recorded by the participants, and mid-sleep time was estimated based on the two sleep indicators.

Results: Most indicators of sleep timing were negatively associated with moderate-tovigorous-intensity physical activity (unstandardized coefficient [B]-0.07 for bedtime and mid-sleep time, $\mathrm{p}<0.05$ ) and daily steps ( $\mathrm{B}$ ranged from -15.51 for bedtime to -13.73 for wake time, $\mathrm{p}<0.05$ ). No associations of sleep timing were found in sedentary behavior and light-intensity physical activity.

Conclusion: The findings suggest that sleep timing should be considered when designing promising strategies or interventions for older women to be physically active.

Keywords: sleep timing, circadian clock, modifiable behavior, aging population

\section{Introduction}

Given that the population of older adults aged 60 years or above is estimated to reach two billion worldwide by $2050,{ }^{1}$ the aging population thus demands a comprehensive public-health response. Physical inactivity (ie, insufficient physical activity [PA]) is identified as the risk factor of death ${ }^{2}$ and is prevalent in older adults. ${ }^{3}$ Considering the components of the 24-hour model are organized into sleep, sedentary behavior (SB), light-intensity physical activity (LPA), and moderate-tovigorous-intensity physical activity (MVPA), ${ }^{4}$ sleep behaviors may be related to the other components and give an insight into improvement in physical inactivity.

Buysse (2014) suggested that the timing of sleep occurring is among the important indicators of sleep behavior; ${ }^{5}$ it is associated with a number of health outcomes, ${ }^{6}$ which may also have influences on behavioral factors in awake time. ${ }^{5}$ A previous study showed that later sleep timing was associated with less time spent on PA and more time spent on SB in adults aged $30-45$ years. ${ }^{7}$ However, 
sleep structure changed across the aging process. ${ }^{8}$ In particular, research has shown that menopause status is associated with sleep alterations ${ }^{9}$ and sleep disorders ${ }^{10,11}$ in older postmenopausal women, indicating the biological change may impact the pattern of sleep timing among women in their older adulthood. Additionally, older women engaged in more sedentary and inactive life patterns compared with older men. ${ }^{12}$ Since older women had shifted longer expected years living with a disability than older men over the past few decades, ${ }^{13}$ the improvement of older women's inactive and sedentary lifestyle is worth our attention. Therefore, investigating the relationship of sleep timing with PA and SB among older women was needed. We aimed to examine the association of sleep timing with SB and different levels of PA (ie, LPA, MVPA, and daily steps) in older women.

\section{Methods}

\section{Study Design}

We used local advertisements and broadcasts to randomly recruit older adults who voluntarily contacted the investigators or representatives from 28 neighborhoods in Taipei City, Taiwan during April and September in 2018. The inclusion criteria were as follows: (a) women aged 60 or above; (b) being healthy community-dwelling; and (c) able to walk independently. A total of 170 older adults were included initially and asked to answer a self-reported questionnaire. Afterwards, each participant had to wear a triaxial accelerometer (model wGT3X-BT; ActiGraph, Pensacola, FL, USA) on the hip for 24 hours for seven consecutive days, except for the time spent on activities involving water. The data collection should meet the standard of wearing the device at least 10 hours per day in waking time for at least four days, including one or more weekend days. ${ }^{14}$ Each participant was given a log with the accelerometer and asked to record their bedtime and wake time, separately, for seven consecutive days. Detailed procedures were reported in previous studies. ${ }^{15,16}$ Eventually, a total of 90 older women meeting the inclusion standard were included in our final analysis. We conducted this study according to the 1975 Declaration of Helsinki and its subsequent revision. ${ }^{17}$ Each participant included in the present research had finished written informed consent. This study was approved by the Research Ethics Committee of the National Taiwan Normal University (REC number: 201711HM003).

\section{Measurement}

The accelerometer data using 60 -second epochs were used to identify the time spent on SB $(<100$ counts per minute [cpm]), LPA (100-2019 cpm), MVPA ( $\geq 2020 \mathrm{cpm}),{ }^{18}$ and daily steps during the total waking time and were processed using the ActiLife version 6.0 software (Pensacola, FL, USA). Three indicators of sleep timing (bedtime, midsleep time and wake time) were included in the study. The average bedtime and wake time of valid days were assessed using the sleep log. The mid-sleep time was calculated as the midpoint of the length between bedtime and wake time, which was used in previous studies among older adults. ${ }^{19,20}$

We considered a series of covariates based on previous studies regarding sleep, SB, and PA. ${ }^{7,15}$ Demographic characteristics including age, marital status, educational level, and job status were assessed by questionnaire. Participants also answered whether they have a balanced diet based on the dietary guidelines in Taiwan. ${ }^{21}$ Pearson correlation was used to examine the correlations between the study variables to identify the covariates for different outcome variables. We included variables showing significant levels of the p-value of 0.05 and the absolute value of coefficient $\geq 0.20$ in the model. Additionally, identification of sleep duration was to crosscheck the sleeping period through bedtime and wake time, and further identified using the Cole-Kripke algorithm ${ }^{22}$ previously used in the older adult population. ${ }^{23}$ The accelerometer wear time was also included in the model based on previous studies. ${ }^{7,15}$

\section{Statistics}

A priori analysis was conducted to estimate sample size through G*Power version 3.1.9.7. ${ }^{24}$ The results showed that minimum sample size to examine the relationship between sleep timing and PA/SB at 0.80 study power is 43 participants, given $\alpha=0.05$ and effect size $=0.35$. Multiple linear regressions were applied to estimate the associations of three sleep timing indicators with SB, LPA, MVPA, and daily steps. The unstandardized coefficients and their $95 \%$ confidence intervals (CI) were estimated. The values of study variables were used in the regression analyses. Furthermore, Pearson correlation was used to examine the correlations between $\mathrm{SB}$, intensity-specific physical activity (LPA and MVPA), and daily steps Table S1. The statistical analyses of regression models and correlation were conducted using IBM SPSS 23.0 
software (SPSS Inc., IBM, Chicago, IL, USA) with $\mathrm{p}<$ 0.05 set as the significance level.

\section{Results}

A total of 90 older women (mean age: 70.5 years, ranging 63.0-87.0) were included in our study. Most of them were married (56.7\%), without receiving tertiary education $(80.0 \%)$, did not have a full-time job (95.6\%), and had a balanced intake (71.1\%). The mean bedtime, wake time, and mid-sleep time were 22:47 (standard deviation [SD]: $0.84 \mathrm{~h}$ ), 06:12 (SD: 1.24 h), and 02:29 (SD: $0.93 \mathrm{~h}$ ), respectively (Table 1).

In the adjusted model, negative associations were found in most of sleep indicators with MVPA (bedtime: $\mathrm{B}=-0.07$, $95 \%$ confidence interval $[\mathrm{CI}]-0.15$ to 0.00 ; mid-sleep time: $\mathrm{B}=-0.07,95 \% \mathrm{CI}-0.14$ to 0.00 ) and daily steps (bedtime: $\mathrm{B}=-15.51,95 \% \mathrm{CI}-26.67$ to -4.34 ; wake time: $\mathrm{B}=-13.73$, 95\% CI -24.74 to -2.73 ; mid-sleep time: $\mathrm{B}=-14.81,95 \%$ CI -25.98 to -3.65 ) (Table 2). The coefficients refer to the association of one minute of delayed sleep timing per day in changes of minutes of MVPA and counts of daily steps. No

Table I Participants' Sociodemographic Characteristics, Sedentary Behavior, Physical Activity and Sleep Timing

\begin{tabular}{|c|c|c|c|}
\hline \multirow[t]{2}{*}{ Variables } & \multirow[t]{2}{*}{ Category } & \multicolumn{2}{|c|}{ Total Sample $(n=90)$} \\
\hline & & $\mathbf{N}$ & $\%$ \\
\hline \multirow[t]{2}{*}{ Marital status } & Married & 51 & 56.7 \\
\hline & Unmarried & 39 & 43.3 \\
\hline \multirow[t]{2}{*}{ Educational level } & Tertiary education $^{\mathrm{a}}$ & 18 & 20.0 \\
\hline & No tertiary education & 72 & 80.0 \\
\hline \multirow[t]{2}{*}{ Job status } & Full- time job & 4 & 4.4 \\
\hline & Non-full-time job & 86 & 95.6 \\
\hline \multirow[t]{3}{*}{ Balanced diet } & Yes & 64 & 71.1 \\
\hline & No & 26 & 28.9 \\
\hline & Description & Mean & SD \\
\hline Age & Years & 70.5 & 5.4 \\
\hline Sleep duration & Minute per day & 426.4 & 60.0 \\
\hline Accelerometer wear time & Minute per day & 925.3 & 73.6 \\
\hline Sedentary time & Minute per day & 599.1 & 71.6 \\
\hline LPA time & Minute per day & 306.7 & 73.8 \\
\hline MVPA time & Minute per day & 19.5 & 19.2 \\
\hline Daily steps & Counts per day & 7082.1 & $305 I .1$ \\
\hline Bedtime & Clock time & $22: 47$ & $0.84 \mathrm{~h}$ \\
\hline Wake time & Clock time & $06: 12$ & I.24h \\
\hline Mid-sleep time & Clock time & 02:29 & $0.93 \mathrm{~h}$ \\
\hline
\end{tabular}

Note: ${ }^{a}$ Tertiary education indicating a university or college degree or higher. Abbreviations: SD, standard deviation; LPA, light-intensity physical activity; MVPA, moderate-to-vigorous-intensity physical activity. associations were found in the three indicators of sleep timing with SB and LPA.

\section{Discussion}

Our findings showed that in older women, later sleep timing was associated with decreased levels of MVPA and daily step counts, but not related to SB and LPA. These findings are informative to intervention designers aiming to promote older adults being physically active should consider altering their sleep timing.

The current study among older women showed consistent results of identifying later sleep timing to be associated with lower levels of PA, including MVPA and daily steps, while no association was found between sleep time and SB time. In keeping with our results, a previous study among older adults found that those who preferred to sleep later (ie, evening chronotype) had lower levels of physical activity. ${ }^{25}$ Another study showed that later sleep timing was related to less PA time but more SB time in middle-aged adults aged 30-45 years. ${ }^{7}$ There were two potential explanations underlying the associations between sleep timing and MVPA in older women. First, studies have shown that older adults spend more time in a greater intensity of $\mathrm{PA}^{26,27}$ in the daytime rather than at nighttime. Therefore, the later sleep timing may lead to shortened daytime in the following day which could be involved in MVPA and decrease the MVPA time of older women. Second, a previous longitudinal study indicated that older adults with evening chronotype (ie, those preferring to stay up later or sleep later) had decreased MVPA participation. ${ }^{19}$ Furthermore, evening chronotype was associated with higher risks of poor health status, ${ }^{19,25}$ and older adults with poorer health status tended to be less active. ${ }^{28,29}$ Alternatively, the engagement in more MVPA during the daytime may synchronize the biological clock by either physical activity or outdoor light exposure that causes early sleep timing. Since this was a cross-sectional study, there might be bidirectional associations between sleep timing and MVPA. Therefore, researches were suggested to further examine the relationships.

Based on our results, 60 minutes delayed in older women's sleep timing may predict a loss of roughly 4.2 minutes MVPA per day, and the accumulation of a week may reduce around half an hour. The negligible loss of MVPA may accumulate over time as the delaying continues for not just one day or a week potentially implicating the decrease in the level of activity and higher risks of unhealthy outcomes in later life. Particularly, older women tend to spend less time on PA, whereas more time on SB generally; ${ }^{3}$ therefore, the time spent on PA may be 
Table 2 Associations of Three Indicators of Sleep Timing with Time Spent on Sedentary Behavior, Light-Intensity Physical Activity, Moderate-to-Vigorous-Intensity Physical Activity, and Daily Steps

\begin{tabular}{|c|c|c|c|c|c|c|c|c|}
\hline \multirow[t]{2}{*}{ Sleep Timing } & \multicolumn{2}{|c|}{$\mathbf{S B}^{\mathbf{a}}$} & \multicolumn{2}{|c|}{ LPA $^{b}$} & \multicolumn{2}{|c|}{ MVPA $^{c}$} & \multicolumn{2}{|c|}{ Daily Step $^{d}$} \\
\hline & B & $95 \% \mathrm{Cl}$ & B & $95 \% \mathrm{Cl}$ & B & $95 \% \mathrm{Cl}$ & B & $95 \% \mathrm{Cl}$ \\
\hline Bedtime & 0.15 & $(-0.13,0.42)$ & -0.14 & $(-0.4 I, 0.12)$ & -0.07 & $(-0.15,0.00)^{*}$ & $-|5.5|$ & $(-26.67,-4.34)^{* *}$ \\
\hline Wake time & 0.01 & $(-0.26,0.27)$ & -0.01 & $(-0.27,0.25)$ & -0.07 & $(-0.14,0.00)^{\#}$ & -13.73 & $(-24.74,-2.73)^{*}$ \\
\hline Mid-sleep time & 0.08 & $(-0.20,0.35)$ & -0.08 & $(-0.34,0.19)$ & -0.07 & $(-0.14,0.00)^{*}$ & $-|4.8|$ & $(-25.98,-3.65)^{* *}$ \\
\hline
\end{tabular}

Notes: ${ }^{a}$ Adjusted for age, balanced diet, sleep duration, and accelerometer wear time. ${ }^{b}$ Adjusted for educational level, sleep duration, and accelerometer wear time. ${ }^{\mathrm{c}}$ Adjusted for age, educational level, sleep duration, and accelerometer wear time. ${ }^{\mathrm{d}}$ Adjusted for age, sleep duration, and accelerometer wear time. ${ }^{*} \mathrm{p}<0.05$; $* * \mathrm{p}<0.0 \mathrm{I}$; ${ }^{\#} \mathrm{p}<0.10$.

Abbreviations: $\mathrm{Cl}$, confidence interval; SB, sedentary behavior; LPA, light-intensity physical activity; MVPA, moderate-to-vigorous-intensity physical activity.

primarily omitted, while the time spent on SB remained. Of note, the decreased daily steps may potentially be contributed by the reduction in MVPA, in view of their strong correlation (coefficient $=0.83$ ). These findings could urge future studies using prospective design to further identify the relationship between sleep timing and intensity-specific activity, particularly in a population with physically inactive lifestyle.

This study holds the strengths that being one of the limited researches to target the older population, including objectively measured SB, PA for analyses. However, several limitations still should be acknowledged. First, due to the cross-sectional study design, the causal relationships between sleep timing and daily steps cannot be inferred. Second, we calculated the midsleep time as the midpoint of the length between bedtime and wake time. However, a previous study showed that the midpoint of the length between sleep onset and offset time was strongly correlated with dim light melatonin onset. ${ }^{30}$ Future studies examining such associations are suggested to use both calculations of mid-sleep time and compare the differences/ similarities. Third, sleep pattern might be different on scheduled days and free days. However, this potential difference was not taken into account. Besides, several confounding factors such as drug use, comorbidity or seasonal factors (eg day length or temperature $)^{31,32}$ were not considered in this study, which may potentially impact the relationships we discussed. Fourth, since the developed algorithm for sleep timing detection was validated for the wrist-worn rather than the hip-worn device, we used self-reported sleep timing indicators in the present study and subjective biases might be caused. Finally, our results might be limited by neglecting participants' medical conditions or other sleeping problems. Future studies can further explore the association between later sleep timing and PA in the older population by incorporating methods for controlling these potential confounders in analysis.
In conclusion, the results found that later sleep timing was negatively associated with MVPA and daily steps in older women. The findings suggest that sleep timing should be considered while implementing strategies or interventions to promote healthy behaviors such as being physically active for older women.

\section{Ethics}

The Research Ethics Committee of the National Taiwan Normal University approved this study.

\section{Acknowledgments}

Dr Yung Liao received personal grants from the Ministry of Science and Technology, Taiwan (MOST 110-2410-H003 -116). Dr Ming-Chun Hsueh received a personal grant from the Ministry of Science and Technology, Taiwan (MOST 109-2410-H-845 -037 -MY2). This work was supported by the Technology Development Program (S2928639) funded by the Ministry of SMEs and Startups (MSS, Korea). The Ministry of Science and Technology, Taiwan and the Ministry of SMEs and Startups, Korea were not involved in the study design, data collection, analysis, and interpretation or writing of the manuscript.

\section{Disclosure}

The authors report no conflicts of interest in this work. There is no financial interest regarding this research nor is there any other conflict of interest.

\section{References}

1. World Health Organization. Active Ageing: A Policy Framework. World Health Organization; 2002.

2. World Health Organization. Global Recommendations on Physical Activity for Health. World Health Organization; 2010. 
3. Kamimoto LA, Easton AN, Maurice E, Husten CG, Macera CA. Surveillance for five health risks among older adults-United States, 1993-1997. Morb Mortal Wkly Rep. 1999;89-130.

4. Rosenberger ME, Buman MP, Haskell WL, McConnell MV, Carstensen LL. Twenty-four hours of sleep, sedentary behavior, and physical activity with nine wearable devices. Med Sci Sports Exerc. 2016;48(3):457-465. doi:10.1249/MSS.0000000000000778

5. Buysse DJ. Sleep health: can we define it? Does it matter? Sleep. 2014;37(1):9-17. doi:10.5665/sleep.3298

6. Chaput JP, Dutil C, Featherstone R, et al. Sleep timing, sleep consistency, and health in adults: a systematic review. Appl Physiol Nutr Metab. 2020;45(10 (Suppl. 2)):S232-s247. doi:10.1139/apnm-20200032

7. Shechter A, St-Onge MP. Delayed sleep timing is associated with low levels of free-living physical activity in normal sleeping adults. Sleep Med. 2014;15(12):1586-1589. doi:10.1016/j.sleep.2014.07.010

8. Gulia KK, Kumar VM. Sleep disorders in the elderly: a growing challenge. Psychogeriatrics. 2018;18(3):155-165. doi:10.1111/ psyg. 12319

9. Gómez-Santos C, Saura CB, Lucas JAR, Castell P, Madrid JA, Garaulet M. Menopause status is associated with circadian- and sleep-related alterations. Menopause. 2016;23(6):682-690. doi:10.1097/GME.0000000000000612

10. Moline M, Broch L, Zak R. Sleep problems across the life cycle in women. Curr Treat Options Neurol. 2004;6(4):319-330. doi:10.1007| s11940-004-0031-6

11. Zolfaghari S, Yao C, Thompson C, et al. Effects of menopause on sleep quality and sleep disorders: Canadian Longitudinal Study on aging. Menopause. 2020;27(3):295-304. doi:10.1097/GME.000000 0000001462

12. Lee Y-S. Gender differences in physical activity and walking among older adults. $J$ Women Aging. 2005;17(1-2):55-70. doi:10.1300/ J074v17n01_05

13. Freedman VA, Wolf DA, Spillman BC. Disability-free life expectancy over 30 years: a growing female disadvantage in the US population. Am J Public Health. 2016;106(6):1079-1085. doi:10.2105/AJPH.2016.303089

14. Migueles JH, Cadenas-Sanchez C, Ekelund U, et al. Accelerometer data collection and processing criteria to assess physical activity and other outcomes: a systematic review and practical considerations. Sports Med. 2017;47(9):1821-1845. doi:10.1007/s40279-017-0716-0

15. Chen B-I, Hsueh M-C, Rutherford R, Park J-H, Liao Y, Oyeyemi AL. The associations between neighborhood walkability attributes and objectively measured physical activity in older adults. PLoS One. 2019;14(9):e0222268. doi:10.1371/journal.pone.0222268

16. Hsueh M-C, Rutherford R, Chou -C-C, Park J-H, Park H-T, Liao Y. Objectively assessed physical activity patterns and physical function in community-dwelling older adults: a cross-sectional study in Taiwan. BMJ Open. 2020;10(8):e034645. doi:10.1136/bmjopen2019-034645

17. World Medical Association. World Medical Association declaration of Helsinki: ethical principles for medical research involving human subjects. JAMA. 2013;310(20):2191-2194. doi:10.1001/jama.20 13.281053

Nature and Science of Sleep

\section{Publish your work in this journal}

Nature and Science of Sleep is an international, peer-reviewed, open access journal covering all aspects of sleep science and sleep medicine, including the neurophysiology and functions of sleep, the genetics of sleep, sleep and society, biological rhythms, dreaming, sleep disorders and therapy, and strategies to optimize healthy sleep.
18. Troiano RP. Large-scale applications of accelerometers: new frontiers and new questions. Med Sci Sports Exerc. 2007;39(9):1501. doi:10.1097/mss.0b013e318150d42e

19. Didikoglu A, Maharani A, Payton A, Pendleton N, Canal MM. Longitudinal change of sleep timing: association between chronotype and longevity in older adults. Chronobiol Int. 2019;36(9):1285-1300. doi:10.1080/07420528.2019.1641111

20. Brychta RJ, Arnardottir NY, Johannsson E, et al. Influence of day length and physical activity on sleep patterns in older Icelandic men and women. J Clin Sleep Med. 2016;12(2):203-213. doi:10.5664/ jcsm.5486

21. Health Promotion Administration. New Guidelines on Daily Nutrition. Administration HP, editor. Taipei, Taiwan: Health Promotion Administration; 2018:6-11.

22. Cole RJ, Kripke DF, Gruen W, Mullaney DJ, Gillin JC. Automatic sleep/wake identification from wrist activity. Sleep. 1992;15 (5):461-469. doi:10.1093/sleep/15.5.461

23. Kim M, Yoshida H, Sasai H, Kojima N, Kim H. Association between objectively measured sleep quality and physical function among community-dwelling oldest old Japanese: a cross-sectional study. Geriatr Gerontol Int. 2015;15(8):1040-1048. doi:10.1111/ggi.12396

24. Faul F, Erdfelder E, Buchner A, Lang AG. Statistical power analyses using G*Power 3.1: tests for correlation and regression analyses. Behav Res Methods. 2009;41(4):1149-1160. doi:10.3758/ BRM.41.4.1149

25. Suh S, Yang HC, Kim N, et al. Chronotype differences in health behaviors and health-related quality of life: a Population-Based Study among aged and older adults. Behav Sleep Med. 2017;15(5):361-376. doi:10.1080/15402002.2016.1141768

26. Davis MG, Fox KR, Hillsdon M, Sharp DJ, Coulson JC, Thompson JL. Objectively measured physical activity in a diverse sample of older urban UK adults. Med Sci Sports Exerc. 2011;43 (4):647-654. doi:10.1249/MSS.0b013e3181f36196

27. Copeland JL, Esliger DW. Accelerometer assessment of physical activity in active, healthy older adults. $J$ Aging Phys Act. 2009;17 (1):17-30. doi:10.1123/japa.17.1.17

28. Moschny A, Platen P, Klaaßen-Mielke R, Trampisch U, Hinrichs T. Barriers to physical activity in older adults in Germany: a Cross-Sectional Study. Int J Behav Nutr Phys Act. 2011;8(1):121. doi:10.1186/1479-5868-8-121

29. Schutzer KA, Graves BS. Barriers and motivations to exercise in older adults. Prev Med. 2004;39(5):1056-1061. doi:10.1016/j. ypmed.2004.04.003

30. Martin SK, Eastman CI. Sleep logs of young adults with self-selected sleep times predict the dim light melatonin onset. Chronobiol Int. 2002;19(4):695-707. doi:10.1081/CBI-120006080

31. Tucker P, Gilliland J. The effect of season and weather on physical activity: a systematic review. Public Health. 2007;121(12):909-922. doi:10.1016/j.puhe.2007.04.009

32. Allebrandt KV, Teder-Laving M, Kantermann T, et al. Chronotype and sleep duration: the influence of season of assessment. Chronobiol Int. 2014;31(5):731-740. doi:10.3109/07420528.2014.901347
The manuscript management system is completely online and includes a very quick and fair peer-review system, which is all easy to use. Visit http://www.dovepress.com/testimonials.php to read real quotes from published authors. 